\title{
Ha ancora senso fare emodialisi domiciliare nel 2001?
}

\author{
Francesco Quarello, Marco Pozzato
}

\section{Divisione di Nefrologia e Dialisi, Ospedale San Giovanni Bosco, Torino}

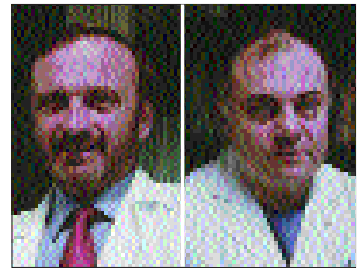

Francesco Quarello e Marco Pozzato
D iprendendo le parole del nostro maestro, il prof. Antonio Vercellone, che quindici anni fa affermava che «nessuno dei radicali vantaggi della dialisi domiciliare è venuto meno a distanza di tredici anni dalla sua introduzione in Piemonte e che, soprattutto a domicilio, dializzano individui e non pazienti», riteniamo che sia opportuno interrogarci se abbia ancora senso nel 2001 investire risorse umane ed economiche a favore di un trattamento che negli anni ha perso sempre più terreno (Figg. 1,2).

Nella nostra Divisione la dialisi domiciliare è sempre stata considerata un trattamento insostituibile per il recupero sociale e riabilitativo del paziente e per il contenimento dei costi, a tal punto che negli anni '70 e '80 la percentuale di pazienti in trattamento aveva raggiunto nel nostro Centro il $38 \%$ dei trattamenti sostitutivi. Nel 2000 la percentuale era del $9.7 \%$, molto bassa se confrontata con quelle della dialisi peritoneale (PD 30.9\%), del Centro ad assistenza limitata
(CAL 13.7\%) e dell'emodialisi ospedaliera (HD 45.7\%), elevata, se paragonata alle percentuali dell'Italia $(1.1 \%) \mathrm{e}$ del Piemonte $(2.2 \%$ ) (Figg. 3, 4).

In effetti, da sempre l'obiettivo del nostro Centro è quello di coinvolgere i pazienti nella conduzione del proprio trattamento; anche quelli in dialisi ospedaliera ricevono informazioni sulla patologia che li ha portati alla dialisi e sulla dinamica della depurazione extracorporea, con il risultato di una buona collaborazione con il personale medico e paramedico.

Se analizziamo però l'età media dei nuovi ingressi in dialisi nell'ultimo decennio, ci rendiamo conto che, a fronte di un'incidenza di 140 pazienti per milione di abitanti in Piemonte, il 54.9\% ha un'età media superiore a 65 anni, con la presenza di almeno 2 fattori comorbidi. Questo fenomeno ha rappresentato uno dei motivi principali dell'abbandono della dialisi domiciliare e di quella ad assistenza limitata. Anche l'età media dei pazienti domiciliari è progressivamente aumentata negli anni, passando da 44 a 49 anni (Fig. 5).

Il nostro intervento nel periodo predialitico è rivolto ai giovani che svolgono un'attività lavorativa a tempo pieno e cercano di mantenere rapporti sociali normali. Una sensibilizzazione precoce serve a far comprendere

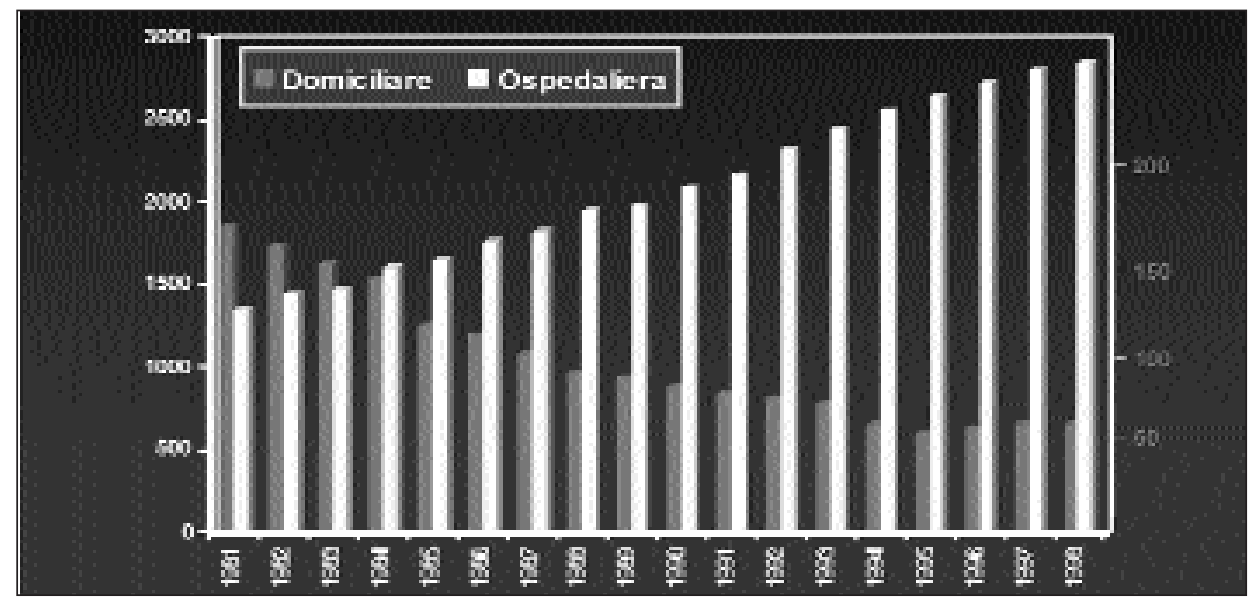

Fig. 1 - Pazienti prevalenti in emodialisi ospedaliera e domiciliare (Piemonte 1981-1998). 
come sia meglio organizzare la dialisi a proprio vantaggio, piuttosto che la vita in funzione della dialisi. Solo così il trattamento dialitico può essere meglio accettato e considerato come una situazione "ponte", che permetta di sopravvivere senza modificare i ritmi quotidiani, in vista di un trapianto renale, che in Italia continua a essere un obiettivo lontano.

Un aspetto da non sottovalutare è quello economico. Da sempre il costo maggiore spetta all'emodialisi ospedaliera, seguita da quella ad assistenza limitata e dalla dialisi peritoneale automatizzata. La dialisi domiciliare è certamente più vantaggiosa, anche se meno della CAPD.

Nel nostro Centro è stato attivato nel 1997, e rinnovato nel 2000, un service globale, con fornitura onnicomprensiva di tutti i materiali, che ha reso molto snelle le procedure d'invio dei nuovi pazienti a domicilio. Nell'ambito di questo servizio è compreso il monitoraggio telematico delle sedute, che sarà attivato non appena disponibile il sistema di raccolta dati del Centro ospedaliero.

Al 31 dicembre 2000 erano trattati a domicilio 20 pazienti $(16 \mathrm{M}, 4 \mathrm{~F})$, con età media di $47.3 \pm 14.2$ anni ed età dialitica media di 79.1 mesi (range 1359). Cinque di essi erano in dialisi giornaliera di 2.30 ore per sei giorni la settimana. Dopo 24 anni di dialisi due pazienti "difficili", affetti da cardiomiopatia dilatativa, hanno finalmente trovato con questo schema un trattamento ben tollerato, che dura da oltre 5 anni. Altri tre pazienti che avevano inizialmente scelto questo tipo di dialisi per motivi di lavoro, lo hanno poi continuato per un evidente miglioramento dello stato di benessere, un miglior controllo della pressione arteriosa e del bilancio calcio/fosforo, una buona correzione dell'anemia, un minor numero di ospedalizzazioni. In questi pazienti (4 su 5 portatori di FAV nativa, 1 su 5 portatore di catetere venoso centrale) il più frequente impiego dell'accesso vascolare non ha comportato inconvenienti di rilievo.

In un paziente che aveva esaurito il patrimonio venoso è stato impiantato il port sottocutaneo Dialock (Biolink

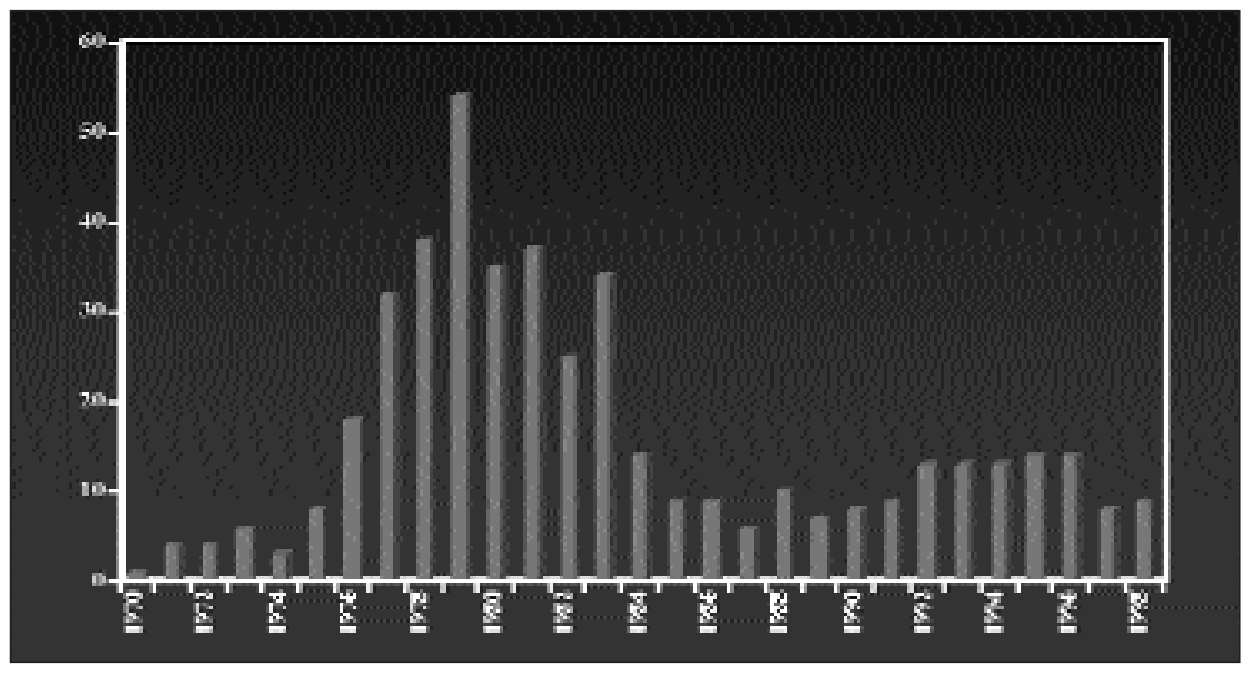

Fig. 2 - Pazienti incidenti in emodialisi domiciliare (Piemonte 1981-1999).

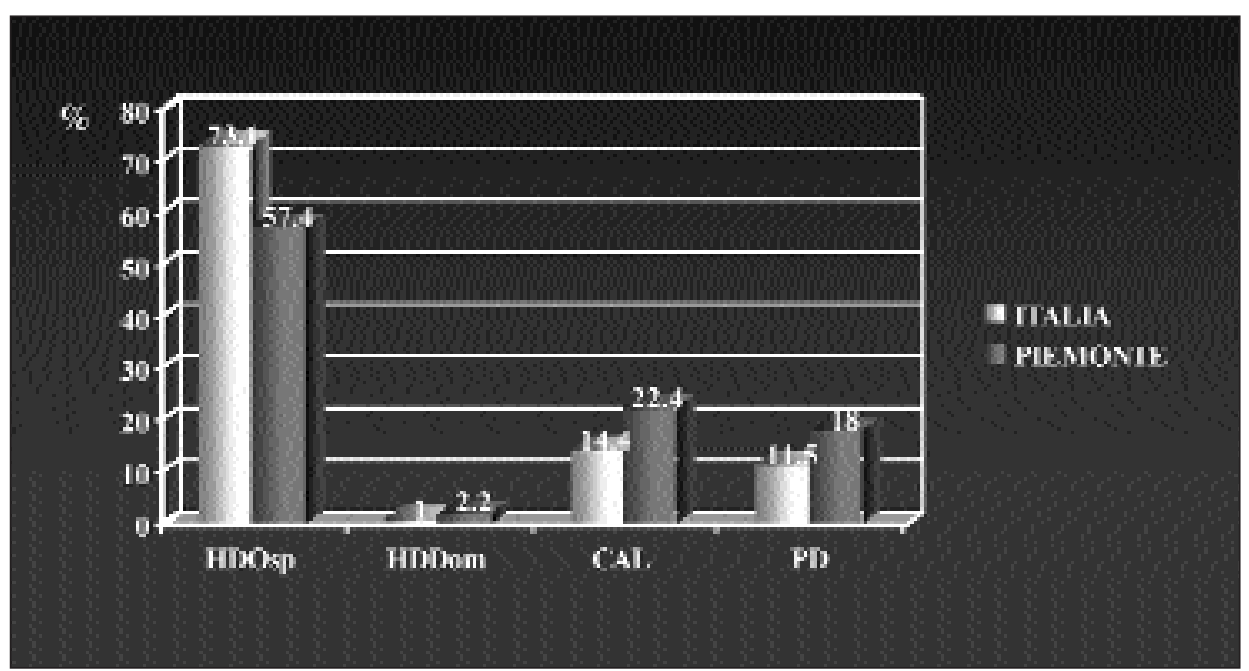

Fig. 3 - Prevalenza dei pazienti in dialisi al 31/12/2000.

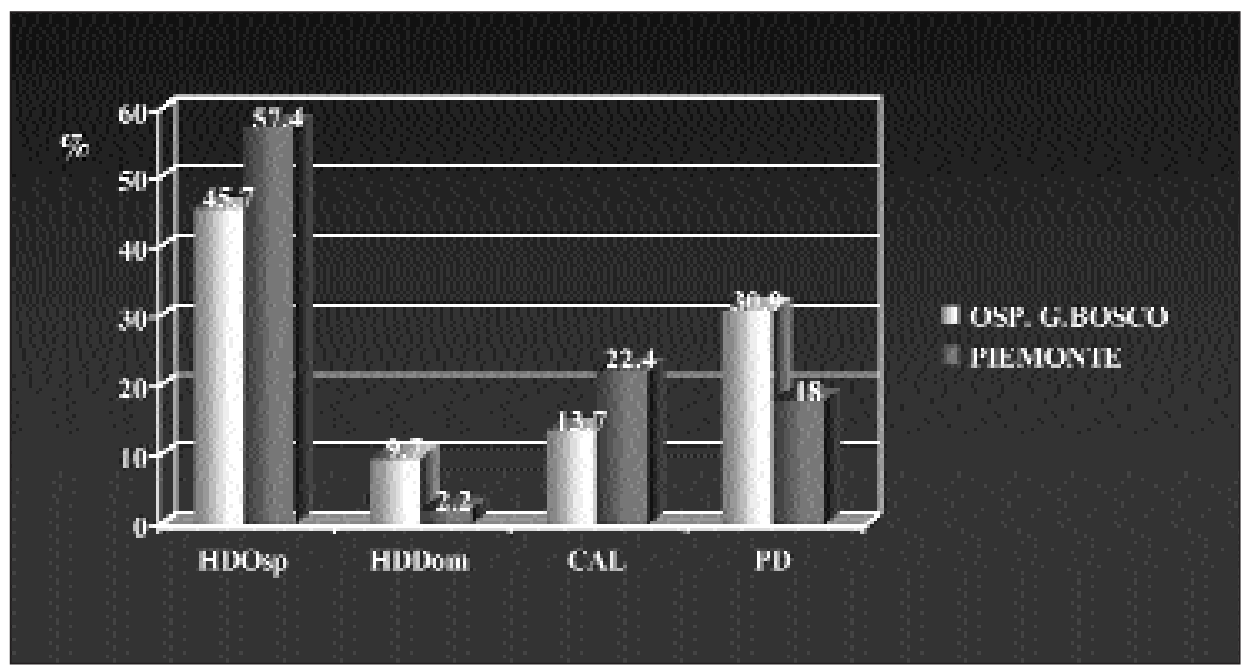

Fig. 4 - Prevalenza dei pazienti in dialisi al 31/12/2000 


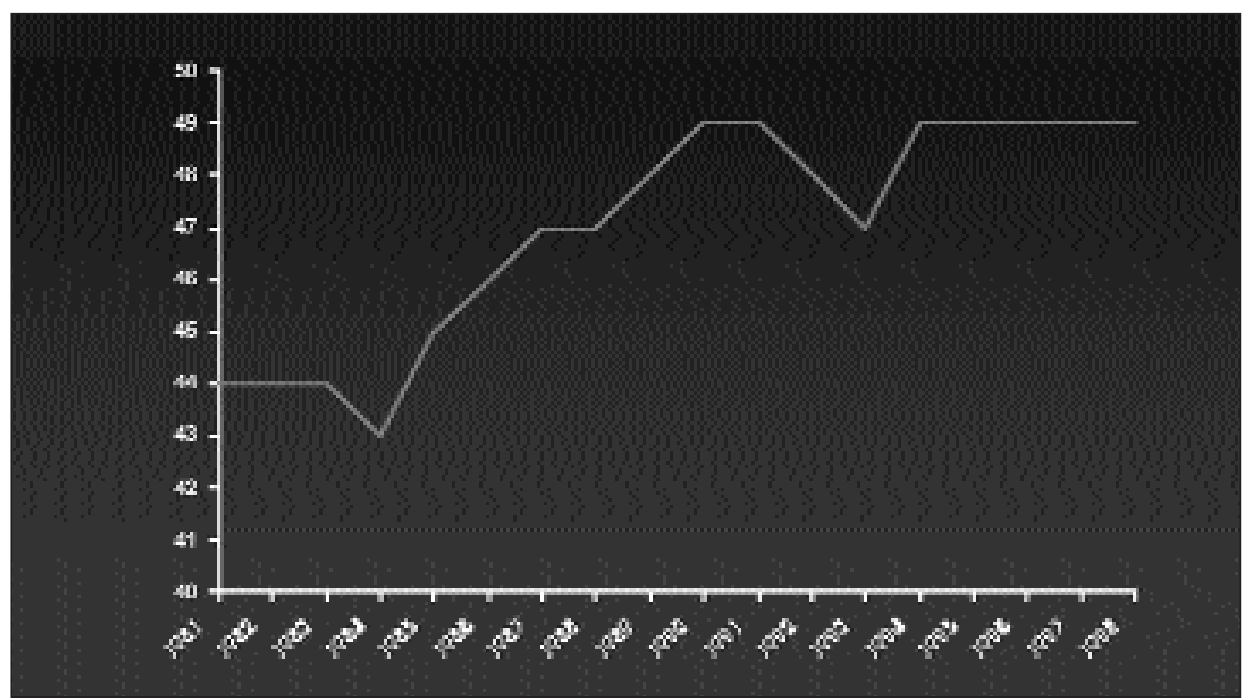

Fig. 5 - Età media dei pazienti avviati all'emodialisi domiciliare (Piemonte 1981-1998).

Corporation). Dopo un breve periodo di addestramento la consorte è stata in grado di utilizzare agevolmente il nuovo accesso con rapido rientro a domicilio.

È stato possibile adottare questi ritmi dialitici personalizzati e utilizzare accessi vascolari insoliti solo grazie alla perfetta collaborazione fornita al medico dal personale dedito all'addestramento. La disponibilità del personale ad adattare i turni lavorativi alle esigenze dei pazienti ha permesso un apprendimento veloce, con una permanenza media in training di quattro-sei mesi. Durante l'addestramento i pazienti eseguono gli esami richiesti per l'inserimento in lista attiva di trapianto renale, col risultato che all'avvio del trattamento domiciliare il $90 \%$ di essi risulta idoneo al trapianto.

Queste considerazioni confermano la convinzione che la dialisi domiciliare debba essere proposta a tutti i pazienti in grado di gestire il proprio trattamento, con il vantaggio di sfoltire le nostre sovraffollate sale dialisi ospedaliere. La carenza di personale è poi un ulteriore stimolo a sviluppare la dialisi extraospedaliera: cinque-sei pazienti inviati a domicilio vogliono dire un infermiere in più nel Centro dialisi ospedaliero.

A parte le considerazioni di ordine economico e organizzativo, il livello di benessere raggiunto dai pazienti è di per sé una valida giustificazione all'apertura di un programma di dialisi domiciliare in ogni Centro ospedaliero, soluzione che appare però improponibile. Il potenziamento di quelli che attualmente lo stanno conducendo con buoni risultati sembra essere un'alternativa ragionevole.

È opinione di chi scrive che l'emodialisi domiciliare debba continuare non solo a esistere, ma anche a ricevere nuova energia da questi scambi di opinione tra i nefrologi italiani. Facciamo in modo che il 2001 rappresenti un punto di partenza, e non di arrivo, di quella pionieristica tecnica tanto cara ai nostri maestri!

\section{fquarello@pobox.com marco.pozzatol@tin.it}

